\title{
Archéopages
}

Archéopages

Archéologie et société

41 | 10/2014-01/2015

La place du cheval

\section{D'une forge imposante à une écurie de la Renaissance au château de Caen}

Évolution d'un édifice lié au cheval entre le XIV et le XVI ${ }^{\mathrm{e}}$ siècle

From an large forge to Renaissance stables at the Castle of Caen. Structural

history and the use of horses between the fourteenth and sixteenth century $A D$

De una imponente herrería a una caballeriza del Renacimiento en el castillo de

Caen. Evolución de una edificación relacionada con el caballo entre los siglos XIV $y X V I$

Bénédicte Guillot et Irène Béguier

\section{(2) OpenEdition}

\section{Journals}

Édition électronique

URL : https://journals.openedition.org/archeopages/979

DOI : 10.4000 /archeopages. 979

ISSN : 2269-9872

Éditeur

INRAP - Institut national de recherches archéologiques préventives

Édition imprimée

Date de publication : 1 août 2015

Pagination : 40-49

ISSN : 1622-8545

\section{Référence électronique}

Bénédicte Guillot et Irène Béguier, « D'une forge imposante à une écurie de la Renaissance au château de Caen », Archéopages [En ligne], 41 | 10/2014-01/2015, mis en ligne le 01 janvier 2017, consulté le 21 janvier 2022. URL : http://journals.openedition.org/archeopages/979; DOI : https://doi.org/10.4000/ archeopages.979 


\title{
D'une forge imposante à une écurie de la Renaissance au château de Caen Évolution d'un édifice lié au cheval entre le XIV et le XVl ${ }^{e}$ siècle
}

\author{
Bénédicte Guillot trara, UMR 6273 “CRAHAM,
}

Avec la collaboration d'Irène Béguier conseil général du Calvados
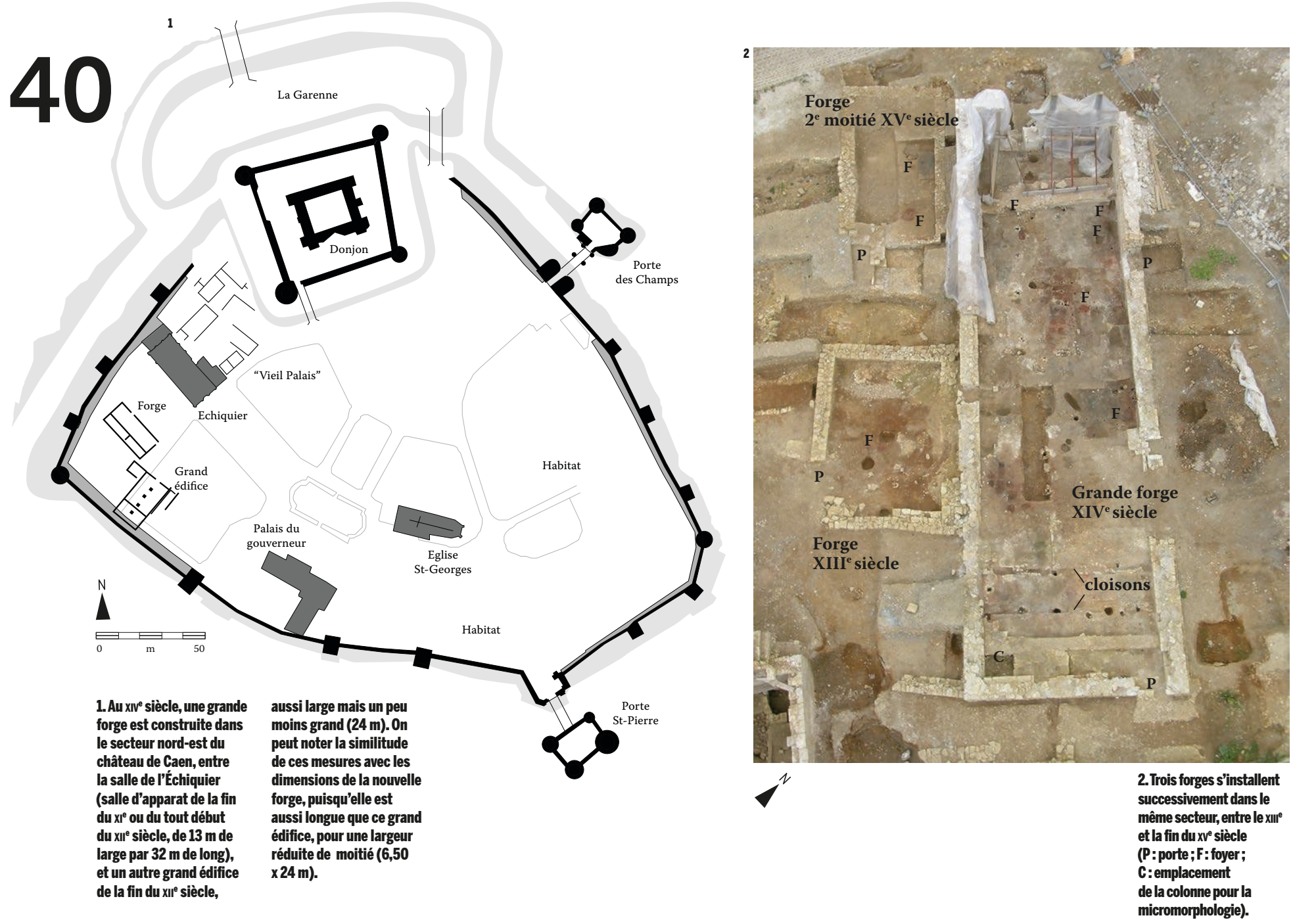
Une fouille menée en 2005 dans l'angle nordouest du château de Caen a permis d'étudier l'évolution de ce secteur de la fortification, dévolu principalement au travail du fer, entre le $\mathrm{XII}^{\mathrm{e}}$ et le $\mathrm{XVI}^{\mathrm{e}}$ siècle ${ }^{\mathbf{1}}$ (Guillot, 2006 ; Guillot, 2015). Une première forge, de dimensions « classiques » pour une installation castrale $\left(30 \mathrm{~m}^{2}\right)$, est bâtie au $\mathrm{XIII}^{\mathrm{e}}$ siècle. Elle est remplacée au XIV ${ }^{\mathrm{e}}$ siècle par un atelier exceptionnel, en premier lieu par ses dimensions, plus de $150 \mathrm{~m}^{2}$, qui ne peuvent être rapprochées d'aucune autre forge castrale connue en Europe. L'explication de cette construction est d'abord à chercher dans les événements politiques jalonnant la guerre de Cent $\mathrm{Ans}^{2}$. Malgré la création de nouveaux foyers dans la première forge, l'augmentation rapide des besoins liés aux activités militaires a dû nécessiter une réorganisation de la forge du château. L'édification d'un grand bâtiment permet de disposer d'un secteur voué à la maréchalerie, d'un autre peut-être dévolu au cerclage des roues; au moins cinq foyers pouvaient fonctionner en même temps. Au XVI ${ }^{\mathrm{e}}$ siècle, le bâtiment est converti en écurie. La fouille a révélé le décor de cette écurie, composé de représentations de mors de chevaux et d'un monogramme, dont l'étude a permis d'identifier le commanditaire des peintures.

\section{L'organisation spatiale et fonctionnelle de la forge du XIV siècle}

La grande forge est élaborée afin d'optimiser toutes les étapes de fabrication des objets. Un bascôté, servant à stocker les matières premières et les matériaux nécessaires au bon fonctionnement de la forge, flanque ce grand bâtiment à l'ouest.

Alors que ce bas-côté est bâti en pans de bois et torchis sur solin en pierre, avec des murs recouverts d'enduits et une toiture réalisée en ardoise de couleur verte et bleu-gris, la grande forge est construite uniquement en pierre de taille calcaire. La taille et le mode de construction de ce bâtiment doivent être mis en relation avec son environnement proche [ill. 1 et 2]. Bien que rendue nécessaire par la guerre, la grande forge fait donc partie d'un programme de construction qui renforce le côté monumental et ostentatoire du château et de l'espace palatial. C'est en effet également au XIV ${ }^{\mathrm{e}}$ siècle qu'apparaît dans les textes le manoir du roi, aujourd'hui musée de Normandie, qui se situe à environ $55 \mathrm{~m}$ au sud du grand édifice, complétant ainsi l'ensemble de grands bâtiments en pierre de taille directement en lien avec le pouvoir politique, militaire et administratif du château.

La forge possède deux entrées larges d'1,10 à $1,20 \mathrm{~m}$ : l'accès principal se situe au sud, dans l'angle sud-est du pignon, tandis qu'une entrée secondaire à l'est, dans le tiers nord du mur gouttereau, permet d'accéder directement dans le secteur de forge proprement dit.

Cinq foyers sont installés au nord et au nord-est du bâtiment, la plupart le long du mur gouttereau afin de profiter de la maçonnerie pour stocker et diffuser la chaleur. Ces foyers, constitués d'argile, sont soit posés directement sur le sol, soit, dans un cas, installés dans une petite cuvette. La répartition des battitures sur le sol a permis de localiser les billots d'enclumes, dont il ne restait plus que les fosses, à moins d'1,50 m des foyers.

La plus grande partie de la forge est installée sur la partie supérieure du terrain naturel, avec par endroits l'ajout de poches de calcaire destinées à niveler le terrain, sauf dans le secteur nord-ouest où le sol est plus soigneusement aménagé, avec la pose de graviers étalés afin de former une assise stable (et par endroits une recharge en mortier). Cet espace pourrait servir de niveau de circulation. Dans la partie centrale, côté est, un aplat de calcaire présente des délimitations spécifiques : il semble consolider le sol autour d'au moins trois structures rectangulaires dont il ne subsiste que le négatif $(0,44 \mathrm{~m} \times 0,38 \mathrm{~m})$. La présence de battitures aux abords, alors que l'on est assez éloigné des foyers, laisse penser à une structure liée à un travail spécifique de forge. Ce pourrait être, par exemple, des supports pour l'embattage des roues. Cette opération nécessite des socles régulièrement espacés afin de pouvoir poser la roue sur une base et la cercler. Il pourrait également s'agir d'un ou de plusieurs établis.

Dès l'origine, le quart sud du bâtiment possède une occupation distincte du reste de la forge : deux tranchées parallèles permettent de restituer deux cloisons orientées est-ouest. Dans la première des tranchées, de petits blocs calcaires servent de calage à une sablière basse (largeur : $0,15 \mathrm{~m}$ ). L'ouverture dans la cloison est matérialisée par un petit élargissement à l'ouest, une interruption des pierres de calage sur 0,90 $\mathrm{m}$ et, surtout, l'apport d'un aplat de mortier marquant le seuil. La seconde tranchée fonctionne avec sept trous de poteaux, quatre situés dans la tranchée, les trois autres étant localisés sur son bord sud. On peut restituer à cet endroit une cloison (en planches ?) maintenue entre des poteaux. Il n'a pas été possible de cerner l'ouverture dans cette cloison. Les deux tranchées sont séparées de $1,25 \mathrm{~m}$; la tranchée sud est située à 2,30 $\mathrm{m}$ du pignon sud, et donc de l'entrée sud du bâtiment.

Létude micromorphologique réalisé par I. Béguier permet de préciser le type d'occupation du secteur entre l'entrée principale au sud et la première tranchée [ill. 3, état 1]. Les premiers niveaux installés sur le terrain naturel correspondent à un espace à usage plutôt domestique, matérialisé, d'abord, par une zone de rejets de foyer à usage culinaire, puis par l'aménagement d'un sol en terre bien entretenu au sommet duquel se trouvent piégés quelques esquilles osseuses chauffées, des charbons de bois et une arête de poisson [ill. 3-a]. Par la suite, l'accumulation, sur ce sol en terre, de litières occupées par des chevaux [ill. 3-b], de battitures, de résidus de peau et de corne chauffée et de matériaux brûlés à hautes températures, témoigne 


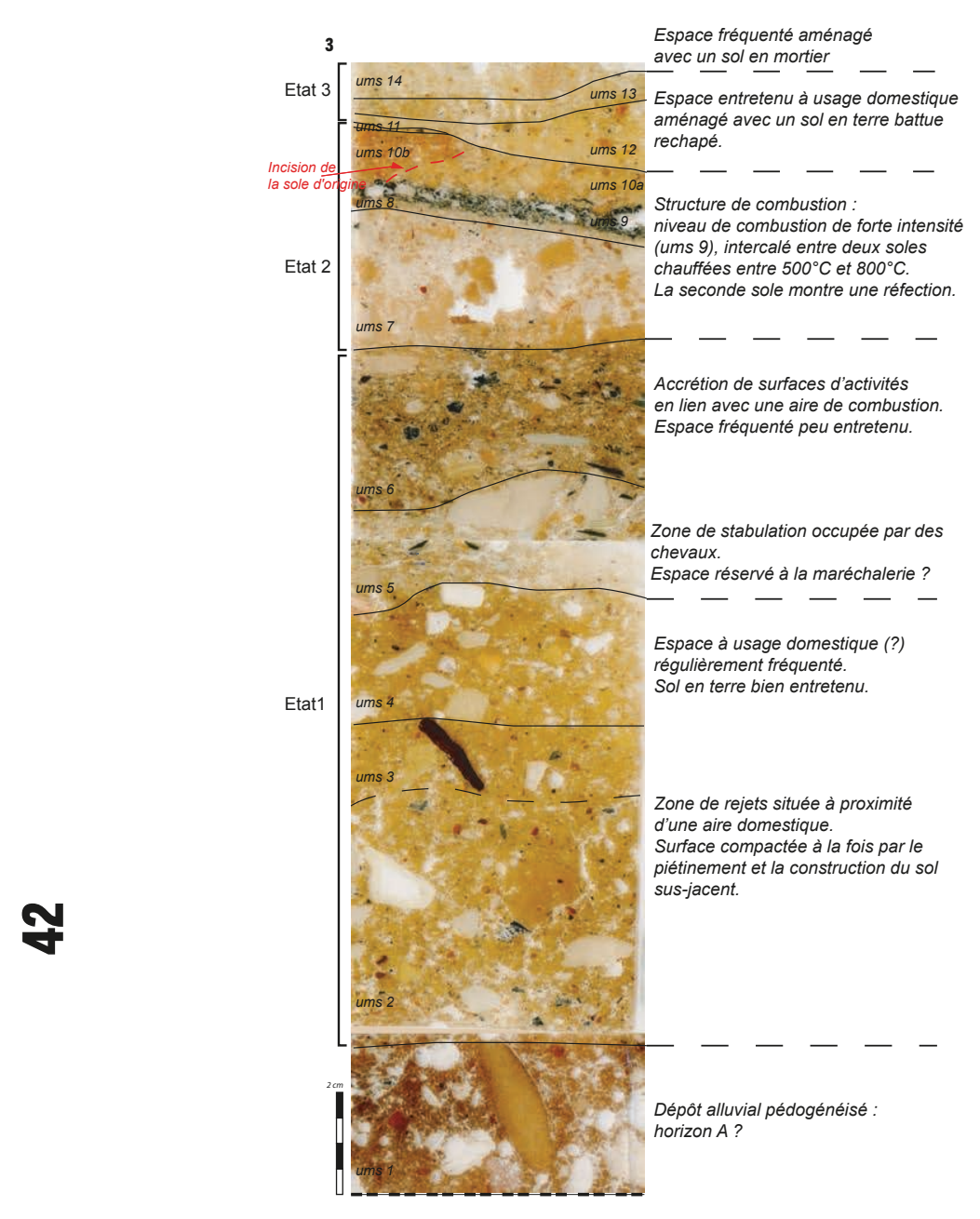

\begin{tabular}{|c|c|}
\hline $\begin{array}{l}\text { 3. La microstratigraphie de } \\
\text { la colonne prélevée au } \\
\text { sud-ouest de la grande } \\
\text { forge (scan de trois lames } \\
\text { minces) permet de } \\
\text { comprendre la nature des } \\
\text { activités qui se sont } \\
\text { déroulées sur place: } \\
\text { photographies des faciès } \\
\text { sédimentaires et des traits } \\
\text { anthropiques observés au } \\
\text { microscope optique: a, } \\
\text { arête de poisson piégée au } \\
\text { sommet d'un sol construit } \\
\text { en terre (ums 4); b, } \\
\text { coprolithe chauffé de } \\
\text { cheval (C) et fine battiture } \\
\text { plate (Bp) observés dans } \\
\text { une zone de stabulation } \\
\text { réservée aux chevaux } \\
\text { (ums 5); c, accrétion de } \\
\text { rejets d'occupation dont } \\
\text { l'abondance,la nature }\end{array}$ & $\begin{array}{l}\text { (charbons [Ch], grains } \\
\text { minérauxu [G] parfois } \\
\text { chauffés) et la distribution } \\
\text { horizontale indiquent un } \\
\text { espace peu entretenu et } \\
\text { régullèrement piétiné,, en } \\
\text { lien avec une activitéde } \\
\text { combustion (ums 6); d, } \\
\text { réfection de sole de foyer: } \\
\text { de facture assez grossière, } \\
\text { la sole d'origine (10a) est } \\
\text { préparée à partirr d'un } \\
\text { sédiment limono-argileux } \\
\text { extraitt d'un horizon illuvial } \\
\text { etd'un dégraissant } \\
\text { minéral constitué de } \\
\text { grains calcaires. Sa } \\
\text { réfection est matérialisée } \\
\text { par une chape brun-rougge } \\
\text { (10b) confectionnée à base } \\
\text { d'un matériau marneux } \\
\text { présent dans la plaine de } \\
\text { Caen (ums 10a et 10b). }\end{array}$ \\
\hline
\end{tabular}
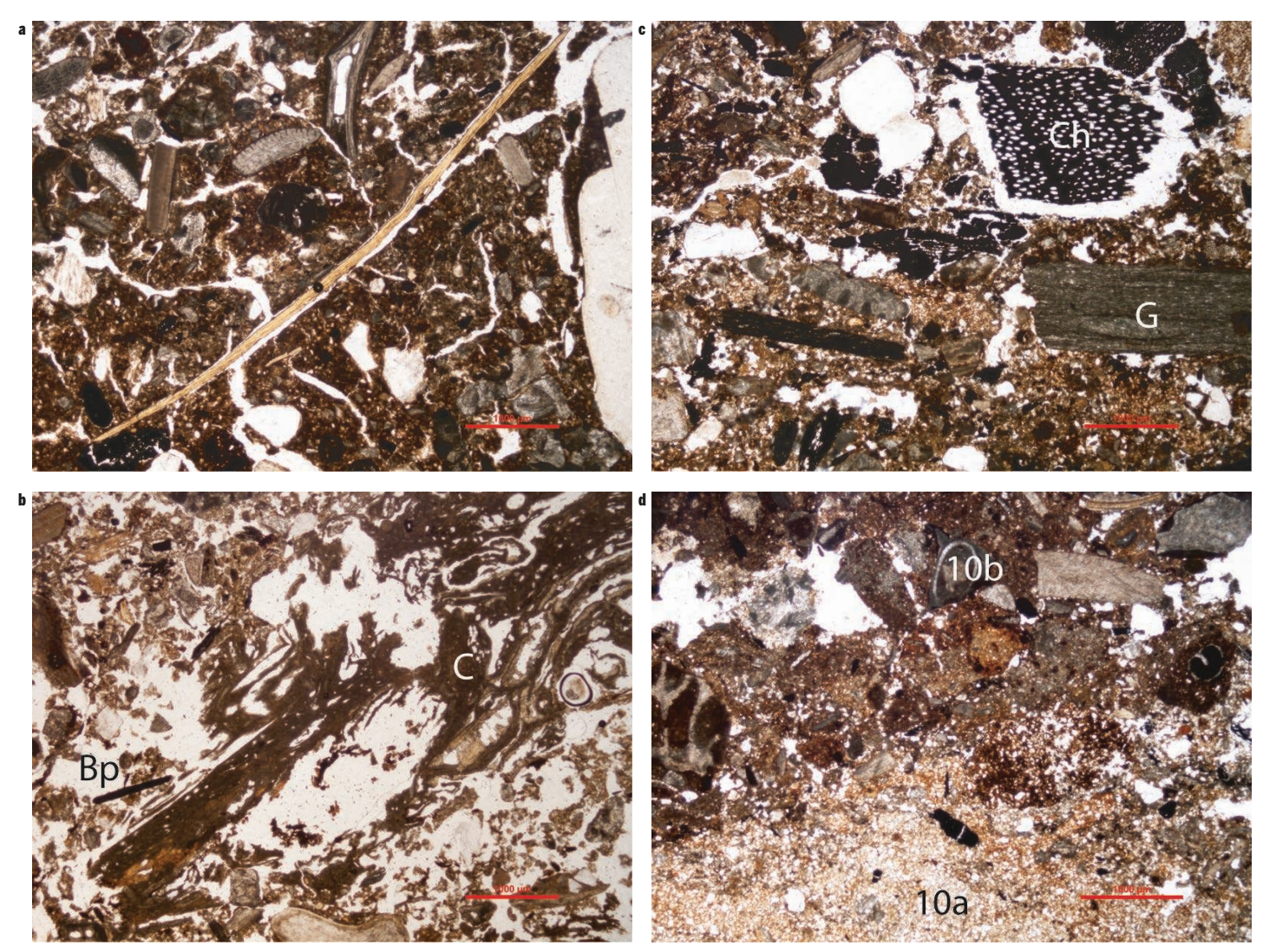
de l'évolution de ce lieu, qui semble dès lors réservé au ferrage des chevaux. Les traces de chauffe, qui marquent aussi bien les résidus de litière que le sommet du sol en terre, peuvent résulter de l'activité de ferrage et/ou correspondre à une combustion légère pratiquée à des fins sanitaires. Aucun foyer n'a été mis en évidence dans ce secteur mais la rubéfaction observée autour d'un trou de poteau pourrait témoigner de cette combustion.

Il semble donc que la partie sud de la forge serve au ferrage des chevaux mais également comme aire domestique pour les forgerons. Il reste à déterminer la destination de l'espace situé entre les deux cloisons, qui ne fait pas plus d'1,25 $\mathrm{m}$ de large. Il pourrait s'agir d'un domaine privé ou d'un lieu de stockage destiné exclusivement à la maréchalerie. Une dernière hypothèse consisterait à restituer à cet endroit un métier à ferrer, constitué d'un bâti très robuste dans lequel le cheval est entravé à l'aide de sangles et de ventrières. Ces métiers à ferrer sont surtout utilisés pour les chevaux qui n'ont pas l'habitude du ferrage (dans le cas contraire, ils restent calmes et le forgeron peut travailler sans crainte de ruades). Il pourrait également s'agir d'un endroit où le maréchal pouvait pratiquer des interventions à caractère hippiatrique (Auliard, 1997).

L'étude paléo-métallurgique montre que cette forge était dévolue à des travaux de finition ${ }^{3}$ (Guillot, 2006 ; Guillot, 2015). Les séquences de travail sont assez courtes, avec de petits coups de marteaux, une intensité de frappe peu importante et un cycle de chauffe limité. Ceci est caractéristique de petites chauffes marquant le stade final du travail d'élaboration des objets en fer, ce qui peut se rapporter, entre autre choses, à la ferrure équestre avec en particulier la mise en forme finale du fer, ou au façonnage d'un fer de trait par exemple. La première forge au XIII ${ }^{\mathrm{e}}$ siècle fabriquait déjà ce type de matériel, mais le nouvel atelier, avec ses cinq foyers, a ainsi pu en fabriquer en plus grand nombre. À l'approche d'Édouard III en 1346, la garnison du château s'élève à 7 à 800 hommes d'armes, 500 archers et 3 ooo miliciens (Prentout, 1904, p. 28). Le besoin en matériel a donc dû être très important et explique en partie l'agrandissement de la forge existante.

\section{L'évolution de la grande forge au début du $\mathrm{Xv}^{\mathrm{e}}$ siècle}

Au début du $\mathrm{Xv}^{\mathrm{e}}$ siècle, le bas-côté de la forge est détruit par un bombardement (lié au siège d'Henri V ?) et, bien qu'elle soit toujours employée pour le travail du fer, il semble que la grande forge soit moins utilisée. Il s'agit toujours d'une forge de finition mais on y travaille aussi les alliages cuivreux, comme le montrent les fragments de ce métal détectés dans les prélèvements, ainsi que les petits éléments de cuivre incrustés dans les parois scoriacées des structures. Les textes nous renseignent sur la période anglaise du château
(1417-1450) et confirment que la ville était sûre et que la garnison n'était pas très importante. Une forge aussi imposante n'était plus nécessaire et on devait $y$ travailler à un rythme beaucoup moins soutenu que durant la phase précédente.

Le fait que Caen ne soit pas un danger pour les forces anglaises est confirmé par un document très intéressant, la " Declaratio hominum armorum, lanceorum et sagittariorum, existentium in castris, fortalitiis, munitionibus, civitatibus et villis in ducatu Normaniae et patria conquesta, etc. ${ }^{\mathbf{4}}$ (British Library, Lambeth Ms. 501 ; Stevenson, 1864, vol. 3, p. 541-542). Ce long texte détaille l'ensemble des garnisons des places fortes de Normandie de la Saint-Michel 1433 à la SaintMichel 1434, en ajoutant, pour quelques-unes, un commentaire expliquant létat de la garnison. On y lit que, sous le commandement du duc de Bedford, la garnison du château de Caen se compose de trois lances montées, vingt-sept hommes à pied et quatre-vingt dix archers, «et non plures quia villa est bene populata et protunc in pacifica patria». C'est ainsi qu'est expliqué le maintien d'un effectif réduit : « et pas plus car la ville est bien peuplée, et maintenant dans une région pacifiée $»^{\mathbf{5}}$.

\section{La garnison : les hommes et les chevaux}

Il est toujours délicat de définir le nombre de chevaux en lien avec une garnison, d'autant qu'il existe plusieurs types de chevaux, parmi lesquels on trouve le destrier, le palefroi, le roncin, le coursier et le cheval de bât, utilisés à des fins différentes.

Au XIII ${ }^{\mathrm{e}}$ siècle, le nombre minimum de montures à la disposition de chaque chevalier est de trois ${ }^{\mathbf{6}}$ mais peut atteindre sept (dans le cas d'un chevalier bachelier), voire dix (pour un baron). Au milieu du XIV ${ }^{e}$ siècle, pour un homme d'armes, on compte deux hommes et deux chevaux (Contamine, 1994, p. 243 et 260). En Italie, à la fin du xve siècle, on compte quatre chevaux par homme d'armes et P. Contamine précise qu'en 1472, dans un projet concernant l’armée milanaise, on atteint le chiffre de sept chevaux par homme d'armes ${ }^{7}$ (Contamine, 1994, p. 244).

Au château de Caen, durant la période anglaise, la garnison est au maximum d'environ 130 personnes ${ }^{8}$. Il faut rappeler que le château de Caen couvre une surface de cinq hectares, avec près d'un kilomètre de courtine. Une garnison de 130 soldats est donc relativement faible pour assurer la défense de la fortification. Si l'on prend le chiffre minimum de trois chevaux par homme d'armes et d'un par archer à cheval, on arrive à un total maximum de près de 150 chevaux en 1429 , sans tenir compte des 51 soldats inscrits sur les montres séparées et dont la catégorie n'est pas marquée. En 1431, sur la centaine de soldats basés à Caen, il faut rajouter une soixantaine d'hommes d'armes et d'archers, tous montés, qui forment une unité mobile nommée «creu » dans les textes. On atteindrait alors un nombre de chevaux aux environs de 200, 


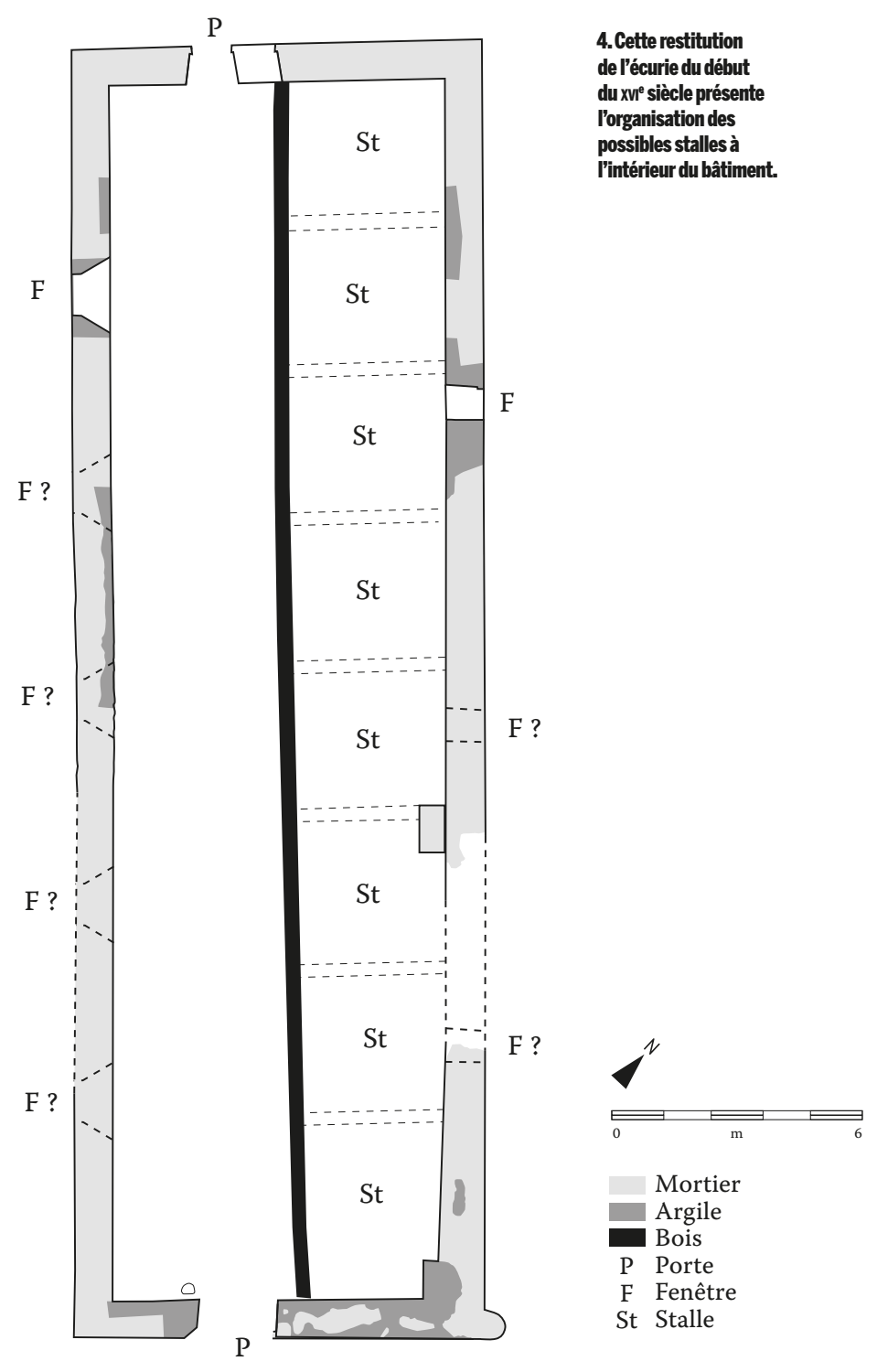

sans qu'il soit possible pour l'instant de savoir où ils étaient logés (au château ?, dans les écuries construites à la fin du XII ${ }^{\mathrm{e}}$ siècle ?). Il s'agit d'une estimation a minima, d'autant plus qu'il faut également prendre en compte les chevaux nécessaires au charroi des marchandises et de l'artillerie, surtout à partir du milieu du $\mathrm{Xv}^{\mathrm{e}}$ siècle.

\section{La fin de la période médiévale}

Après la guerre de Cent Ans, le bâtiment qui abritait la grande forge est reconverti en habitat, avec un cloisonnement de l'espace interne : la cuisine au sud, vers l'entrée, et des espaces plus résidentiels au nord. On aurait ainsi un bâtiment divisé en plusieurs pièces, dont les niveaux de circulation sont composites (graviers mêlés à de petits blocs de calcaire, terre battue, mortier, calcaire) et ne couvrent pas une surface importante.

L'étude micromorphologique permet de préciser l'utilisation de certaines de ces pièces. Au sud-ouest, l'espace est régulièrement fréquenté et aménagé avec des sols en terre finement travaillés et régulièrement entretenus (traces de réfection et de balayage). La présence à leur surface de quelques charbons de bois et fibres végétales, de rares esquilles osseuses chauffées et coquilles d'œufs, évoque une aire domestique en lien avec la préparation et/ou la cuisson des aliments. Au nord-ouest, l'espace est plus faiblement fréquenté mais régulièrement entretenu. Parmi les sols qui se succèdent, un sol en terre, qui a fait l'objet d'un travail particulièrement soigné, repose sur un fin niveau de préparation comportant des résidus de forge et se compose d'un matériau finement trié et soigneusement malaxé. L'attention particulière portée à l'aménagement et à l'entretien de cet espace suggère un lieu situé à l'écart des activités domestiques culinaires. Dans un dernier temps, un changement d'utilisation est révélé par l'installation d'un sol en terre plus grossier, présentant des traces d'un travail domestique proche d'une aire de combustion, peut-être en relation avec des poches de cendre mises en évidence dans la partie nord du bâtiment.

Ce secteur du château est pourtant toujours affecté au travail du fer et une nouvelle forge est construite dans une zone laissée à l'abandon. $\mathrm{Ce}$ 
nouvel atelier reprend des dimensions plus modestes $\left(33 \mathrm{~m}^{2}\right)$, mais reste toujours parmi les ateliers castraux les plus importants recensés en Europe. Le foyer de forge est constitué de pavés calcaires, avec un contrecœur en tuileaux. Un autre foyer, en argile, sert probablement aux activités culinaires. La construction, élevée en torchis-colombages sur solins de pierre, possède des parois internes enduites de mortier de chaux. Des fragments de ces enduits contenaient encore des traces de peinture, formant des bandes de couleur rouge ou verte, sans qu'il soit possible de restituer un décor.

Au tournant du XVI ${ }^{\mathrm{e}}$ siècle, cette nouvelle forge est démolie. Le lieu sert de dépotoir, en particulier pour les déchets de forge, et le grand bâtiment retrouve son activité d'origine. Huit nouveaux foyers en pavés calcaires sont construits et de nombreux forgerons reprennent le travail du fer, toujours à la fin de la chaîne opératoire, dans le cadre d'une forge de finition. Ces nouveaux foyers sont positionnés le long du mur gouttereau oriental, reprenant ainsi le plan de la forge du XIV siècle, mais le cloisonnement au sud n'existe plus.

La remise en fonction de la grande forge est de courte durée puisque, au tout début du XVI ${ }^{\mathrm{e}}$ siècle, l'activité métallurgique est définitivement abandonnée dans ce secteur du château. Elle reviendra plus tard, au XVIII ${ }^{\mathrm{e}}$ siècle, mais ce sera cette fois la salle de l'Échiquier qui servira de forge. Entre temps, le grand bâtiment a été une nouvelle fois reconverti, pour servir de salle d'apparat d'un nouveau genre : une écurie prestigieuse comportant un décor peint unique.

\section{Lécurie de la Renaissance}

C'est le gouverneur du château Jean de Feschal qui semble utiliser la grande bâtisse pour en faire une écurie prestigieuse [ill. 4], en accord avec le goût de l'ornementation du début de la Renaissance. Une grande partie du parement intérieur est refait, les foyers ayant fortement abîmé les pierres. L'apport d'un épais niveau de calcaire permet d'assainir les anciens sols de forge. Deux grandes portes, se faisant face, sont ouvertes au nord et au sud, des fenêtres venant compléter le dispositif dans les murs gouttereaux. Une grande poutre sablière traverse le bâtiment du nord au sud et le divise en deux parties distinctes. Elle forme ainsi un secteur ouest qui comprend les deux seuls accès au bâtiment (large de 3,30 $\mathrm{m}$ au nord et 3,60 $\mathrm{m} \mathrm{au}$ sud), et un secteur oriental moins large (large de 3,12 $\mathrm{m}$ au nord et 2,60 $\mathrm{m}$ au sud), dans lequel on peut restituer des stalles. Enfin, les murs sont recouverts d'enduit, mais seul le côté ouest est décoré par un motif en quinconce. Les peintures représentent différents mors de brides et un monogramme [ill. $5 a$ et b].

Le décor, qui débute à environ 1,20 $\mathrm{m}$ du sol, est présent sur au moins quatre rangées. En extrapolant à partir des enduits conservés, le bâtiment devait présenter environ 140 dessins de mors. La réalisation à main levée de chaque motif et les évidentes différences entre chacun des mors conservés établissent qu'il s'agissait d'un décor unique, résultat d'un programme bien arrêté à l'avance.

Le monogramme offre toujours la même taille, ce qui permet de supposer l'utilisation d'un pochoir. Le motif a la particularité d'être identique si on le retourne. Notons au passage la recherche d'effet polychrome dans la disposition des monogrammes. En effet, ils sont alternativement en noir ou en bleu. Quant aux mors, certains d'entre eux ont été légèrement rehaussés en grisvert, sans doute pour donner plus de relief, mais la grande majorité sont monochromes, ayant été simplement dessinés.

Les mors de chevaux, tous différents, ont été dessinés à l'échelle 1/1 (o,35 m de haut pour o,24 m de large). Si la composition montre une volonté d'ornementation par l'alternance du monogramme et des motifs, ce n'est pas la partie la plus décorée du mors de cheval qui est mise en valeur mais la partie technique, la bride [ill. 6 a-d]. Cette dernière se divise principalement en trois : l'embouchure ou le mors (appelé communément canon), la gourmette et les branches. Les branches sont de deux sortes dans les enduits peints et aucune bossette (ornement en saillie fixé aux deux extrémités du canon) n'est représentée, bien que cet objet participe à l'ornementation d'un mors. L'originalité de ces enduits peints résulte donc non seulement du choix de peindre des mors mais également de s'attacher à une partie qui peut sembler moins esthétique, le canon.

L'étude de ces peintures a été entreprise par Vincent Juhel ${ }^{\mathbf{9}}$ qui les a comparées avec des dessins issus d'un manuscrit écrit par Jean de Feschal, capitaine du château de Caen entre 1504 et 1516. Ce manuscrit sur parchemin nommé «traité d'hippiatrie et d'embouchure ${ }^{\mathbf{1 0}}$ se compose de deux traités. Le premier, le traité d'embouchure, comprend 160 dessins de mors réalisés à l'encre ; le second est un traité d'hippiatrie qui comporte 36 recettes pour soigner les chevaux et n'est pas illustré. Sur les treize mors représentés au château suffisamment complets pour être identifiés, douze peuvent être rapprochés avec certitude du manuscrit de Jean de Feschal ; le treizième ne trouve pas exactement son parallèle dans le traité d'embouchure mais peut être comparé à au moins deux dessins. Les correspondances entre le manuscrit de Jean de Feschal et les peintures conservées permettent de voir dans le décor mural la copie du manuscrit du commanditaire.

Ce type de décor illustre le renouveau de l'art équestre car chaque type de mors de bride y est détaillé selon la nature du cheval, la grandeur de sa bouche, avec la mention de caractère ou du défaut à corriger. Chaque mors correspond à un type de cheval bien distinct. Un point important du traité est le passage dans lequel Jean de Feschal mentionne l'un de ses prédécesseurs, Alain Gouyon, qui 
b. Vue générale des enduits

peints conservés sur

la portion nord du mur

gouttereau ouest.
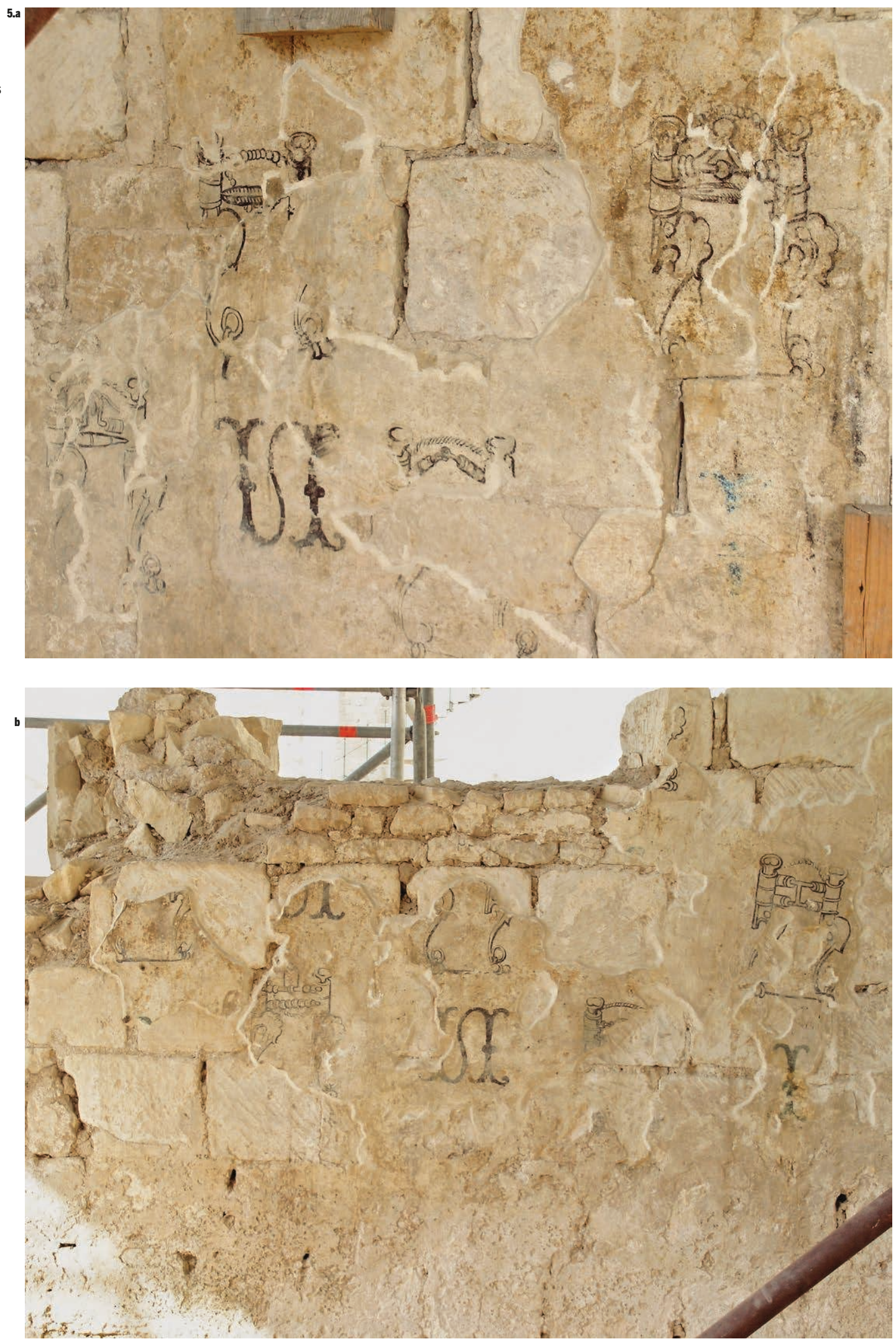
a également travaillé sur les mors de brides, mais s'intéressa plutôt aux aspects techniques et à l'expérimentation. Une hypothèse séduisante, que rien ne peut cependant confirmer ${ }^{\mathbf{1 1}}$, serait de situer dans

la grande forge, lorsqu'elle est utilisée en tant que telle pour la dernière fois, à la fin $\mathrm{du} \mathrm{XV}^{\mathrm{e}}$ siècle, les expérimentations des divers mors de chevaux par le gouverneur du château Alain Gouyon.

Les reproductions grandeur nature de mors déclinées en catalogue pourraient tout d'abord témoigner d'une fonction éducative. Les exercices équestres se développent à partir de la fin du Moyen Âge et constituent dès les siècles antérieurs une part importante de l'éducation des nobles. À partir du milieu du $\mathrm{XVI}^{\mathrm{e}}$ siècle, l'équitation savante devient un des arts majeurs, avec la publication de l'ouvrage de l'écuyer napolitain F. Grison (original de 1550), ouvrage très tôt traduit en français et réédité à de nombreuses reprises. Les peintures du château sont contemporaines des études italiennes sur cette pratique de l'équitation savante du début $\mathrm{du} \mathrm{XVI}^{\mathrm{e}}$ siècle. Si l'on prend en compte l'ensemble des aménagements effectués dans le bâtiment, il semble plus probable que le bâtiment ait servi d'écurie. À l'appui de cette hypothèse, on peut rappeler, outre la nature spécifique des peintures, la répartition en possibles stalles du côté est, là où l'enduit des murs est resté blanc.

Jean de Feschal choisi donc d'adapter un édifice désaffecté pour en faire une écurie castrale évoquant déjà les écuries Renaissance, qui se développent plutôt à la fin du Xvi ${ }^{\mathrm{e}}$ siècle. Les recherches menées par $\mathrm{D}$. Alexandre-Bidon et $\mathrm{P}$. Mane sur « l'iconographie de l'écurie (XIII ${ }^{\mathrm{e}}$-début du XVI ${ }^{\mathrm{e}}$ siècle) $\gg{ }^{\mathbf{1 2}}$ montrent que les écuries représentées possèdent des caractéristiques communes : des ouvertures cintrées et peu larges, avec la possible présence d'une marche pour accéder à l'intérieur; des fenêtres hautes et étroites ; un plafond plat avec le fenil à l'étage ; la présence ou non de stalles, de râteliers ou de mangeoires de toutes sortes. Sans vouloir calquer à tout prix le bâtiment étudié sur les enluminures médiévales, certains de ces caractères se retrouvent néanmoins à Caen. La porte sud est tout juste assez large pour permettre le passage de chevaux $(1,50 \mathrm{~m})^{\mathbf{1 3}}$ et possède une marche. Les deux murs gouttereaux présentent des fenêtres, mais ces dernières ne sont ni alignées, ni semblables. De plus, si on restitue le même écartement pour les ouvertures sur les deux murs gouttereaux, on dénombre trois ou quatre baies à l'ouest et deux seulement à l'est. À l'ouest, côté peintures murales, l'ouverture est ébrasée (1,50 m de largeur côté interne), apparaissant à 1,90 m du sol. En revanche, côté oriental, la baie, qui ne présente aucun ébrasement, est large de o,70 m. Cette dernière pourrait donc être identifiée aux fenêtres étroites et hautes de l'iconographie, qui maintiennent le cheval à l'abri des courants d'air tout en permettant la ventilation.
En outre, le traité d'hippiatrie publié en 1614 par Jean Tacquet (Tacquet, 1614, p. 149-162, cité par Liévaux, 2009, p. 70-72) donne la description d'une écurie : la pièce devra être haute d'au moins 3,70 $\mathrm{m}$ (12 pieds) et "plus est elle haute E plus elle sera belle E saine pour les chevaux si l'air y peut donner $\mathcal{E}$ traverser des deux costez $»$ (Tacquet, 1614, p.160). La présence d'ouvertures sur les deux murs gouttereaux permet cette circulation de l'air. La hauteur du bâtiment de Caen reste inconnue, cependant les maçonneries étaient conservées sur plus de 3,40 $\mathrm{m}$ de haut avec une nouvelle porte de $3 \mathrm{~m}$ de haut percée dans le pignon nord.

La possibilité d'un étage sous comble a déjà été évoquée pour la période précédente, avec la mise au jour, le long du pignon sud, d'un massif maçonné, dont l'arasement a empêché de définir la fonction, peut-être un massif d'escalier. Ce massif est démoli afin de permettre la création de la nouvelle porte sud mais la mise en place d'un autre accès est tout à fait possible. Cet espace au-dessus de l'écurie est recommandé par les traités du XVI ${ }^{\mathrm{e}}$ siècle pour y mettre le fourrage.

Concernant les séparations entre chevaux, la restitution de deux poutres reliant la sablière centrale crée des stalles de 2,60 m de large sur plus de $3 \mathrm{~m}$ de long. Les séparations entre chevaux pourraient être assurées simplement par ces poutres, à moins que ces dernières n'aient servi de support à une clôture formée de planches verticales. Les râteliers et les mangeoires n'ont laissé aucune trace au sol ou sur le mur. Mais il est possible que ces mangeoires aient été disposées sur des tréteaux ou montées sur des pieds fins, qui n'auraient laissé aucun vestige. Il faut remarquer que ces possibles stalles sont très vastes par rapport aux indications données dans les ouvrages d'hippiatrie ; Jean Tacquet propose un espace large d'1,6o m, voire de $2 \mathrm{~m}$ pour les juments (Tacquet, 1614, p. 160), ce qui est déjà plus grand que l'écartement des écuries royales des Tuileries, qui mesure $1,40 \mathrm{~m}$ et qui était considéré au XVI ${ }^{\mathrm{e}}$ siècle comme exemplaire (Liévaux, 2005, p. 73). Selon la synthèse des articles ou traités sur les écuries réalisée par J.-J. Schwien et Y. Jeannin, « les longueurs des stalles oscillent entre 2,45 et $4 \mathrm{~m}$, leur largeur entre 1,35 et 1,75 $\mathrm{m} »$ (Schwien, Jeannin, 2012, p. 122). La largeur des probables stalles de Caen peut être relativisée par le fait que les stalles pouvaient facilement accueillir deux chevaux en fonction des besoins. Si on restitue le même écartement sur toute la longueur, le bâtiment aurait pu contenir au maximum huit stalles, probablement pour les chevaux du gouverneur. Comme le signale A. Salamagne, « la séparation entre les diverses espèces de chevaux, "haquenée" ou jument, "roncin " ou cheval de bât, "coursier", "destrier" ou étalon, palefrois luxueusement caparaçonnés, était courante » (Salamagne, 2010, p. 30).
11. Aucun mobilier

dernière phase

$\mathrm{X}^{\mathrm{e}}$ congrès de la Société

en mai 2012. Je les remercie

communiquer leur

documentation.

13. Cette étroitesse de

dans les écuries du château

du Rivau (Indre-et-Loire)

qui forment pourtant le

principal accès du château (Liévaux, 2005, p. 60, fig. 31) 
6.a.Comparaison du mors

CC avec un exemplaire

du xvie siècle conservé

au Château-musée

de Saumur ; selon

le manuscrit de Jean de

Feschal (pl.XXIII), le mors

est recommandé « A cheval

qui suyt à la main, puis

porte la teste bace

et la bouche ouverte i.

b. Photographie et dessin

du mors $\mathrm{G}$, affecté, dans

le manuscrit de Jean de

Feschal (pI.XXIIv v),

« A cheval qui ne craint

mulz mors, aulcunefois

a la bouche et qui joue

de leschine is.

c. Mors Q pour, selon

le manuscrit de Jean

de Feschal (pl.XXXIVvo'),

" A cheval à la bouche

tendre $)$.

d.Mors LL assigné, selon

le manuscrit de Jean

de Feschal (pl.XXXII),

«A cheval qui prens

le mors aux dens sans

estre eschauffén.
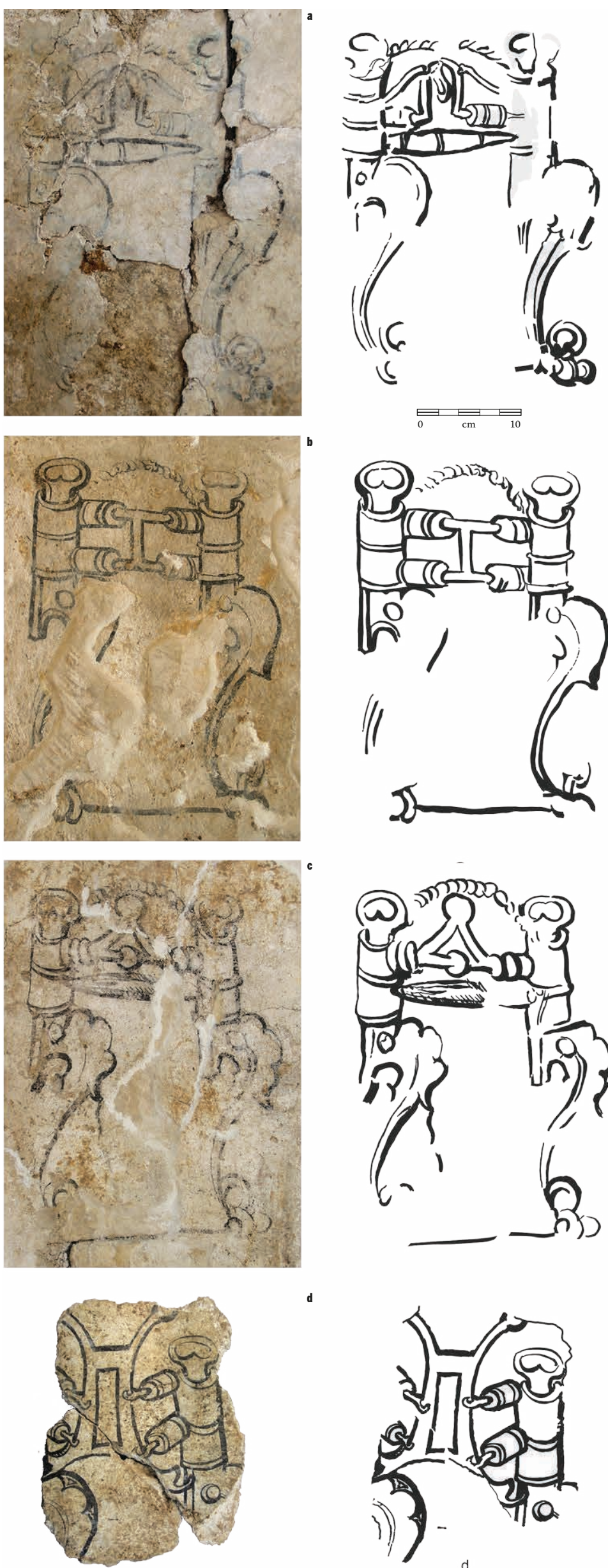

Cuper ace cl
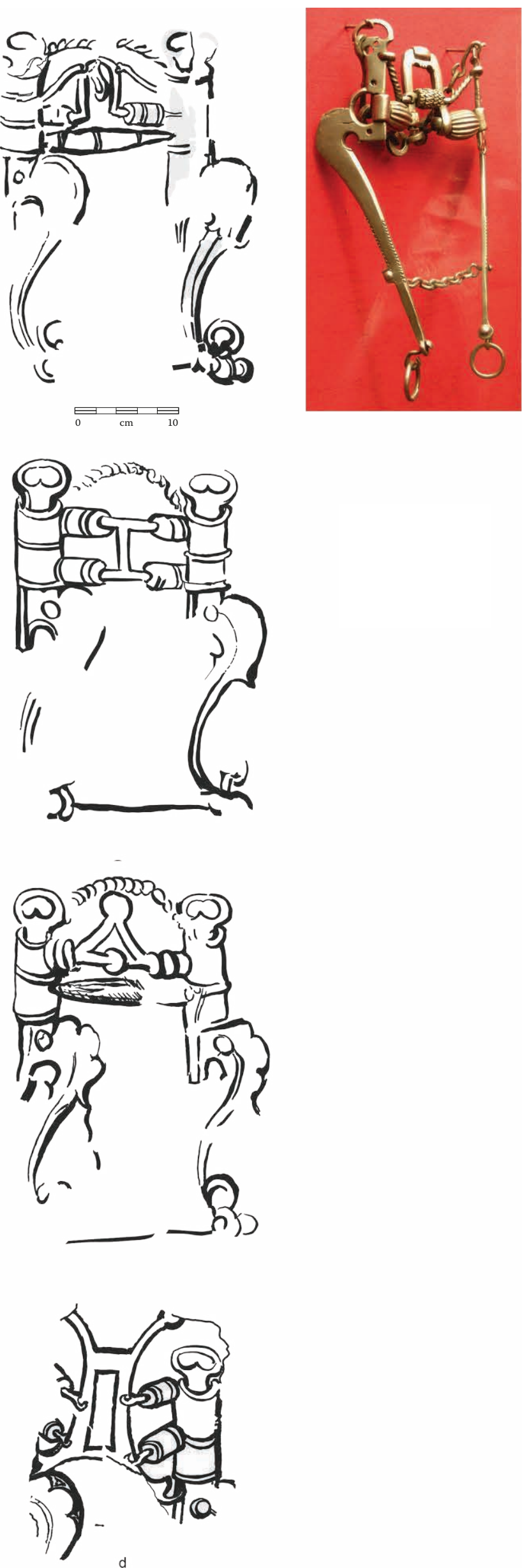
Au tournant du $\mathrm{XVI}^{\mathrm{e}}$ siècle, le statut réel et symbolique du cheval, au centre de la plupart des occupations humaines, se transforme. Comme le signale D. Roche, « le cheval n'est plus une simple monture utilitaire, c'est un emblème dont la beauté et les qualités doivent être mises en valeur », l'écurie devient alors « un espace d'affirmation distinctive » (Roche, 2005, p. 13). Il est significatif que Jean de Feschal, qui transforme l'ancienne forge en écurie de la Renaissance, s'identifie dans son manuscrit comme «chevallier». De plus, il met en exergue de son livre sa devise - « Rien qui ne l'a » - et le nom de sa seigneurie, « Marboué ». L'héritage des chevaliers médiévaux est encore revendiqué par les membres de l'aristocratie. S'intituler chevalier, avant tout autre qualificatif (capitaine du château de Caen ou chambellan du roi), est évocateur : Jean de Feschal écrit son traité en tant que tel, le cheval symbolisant les vertus guerrières et le « vivre noblement » (Liévaux, 2005, p. 22). Le cheval devient alors, selon les termes d'E. Münzberg, « une œuvre d'art. En effet, le cheval n'est pas seulement un produit naturel d'une grande noblesse, il est travaillé par le cavalier et devient par ce processus de transformation une œuvre d'art au service de la représentation princière. Les "chevaux œuvres d'art" sont alors logiquement rassemblés dans les bâtiments de collections que sont les écuries et jouent à la cour un rôle de premier ordre » (Münzberg, 2010, p. 70).
P. Liévaux a recensé les écuries ayant conservé des décors peints. Il note que la pratique des peintures à l'intérieur d'écuries prestigieuses fut certainement plus répandue que ne le laissent supposer les rares vestiges connus (Liévaux, 2005, p. 51). Sont conservés le décor peint des voûtes du château de Villeneuve-Lembron (Puy-de-Dôme), datant des années 1590 (scènes de bataille), quelques silhouettes d'animaux et vestiges de scènes équestres dans les écuries du château des Cars, au sud de Limoges (1515-1520) ; à Creully (Calvados), un cheval en mouvement a été peint sur un des murs de l'écurie du château au début du XVII ${ }^{\mathrm{e}}$ siècle.

Même s'il participe du même mouvement artistique, le décor du château de Caen reste très singulier dans ses motifs ${ }^{\mathbf{1 4}}$. Le choix de représenter un des accessoires spécifiques de l'art équestre, le mors de bride, et non pas le symbole de cet art, le cheval, reflète l'intérêt de Jean de Feschal pour les aspects techniques de l'équitation, plus peut-être que la pratique elle-même. Jean de Feschal a également choisi d'alterner ces mors de bride avec un monogramme, qui ne semble pas pouvoir être raccordé à son nom. Cette marque pourrait être celle qu'il utilisait pour marquer ses chevaux au fer. Le cheval serait alors bien représenté dans le décor, mais au travers de cette marque qui permettrait également de rappeler l'identité du commanditaire de ces peintures.

\section{Références bibliographiques}

STEVENSON J., 1861-1864, Letters and papers illustrative of the wars of the English in France during the reign of Henry the Sixth, king of England, Londres, Longman, Green, Longman, 2 t. en 3 vol.

AULIARD C. (1997), « Les maréchaux à l'époque médiévale : forgerons ou vétérinaires ? ", Médiévales, $n^{\circ} 33$, p. 161-173. En ligne : http://www. persee.fr/web/revues/home/prescript/article/ medi_0751-2708_1997_num_16_33_1403

Contamine P., 1994 (4 éd.), La guerre au Moyen Âge, Paris, Presses universitaires de France, 516 p.

Guillot B., 2006, Caen, le château. Abords de l'enceinte nord. Fouilles de 2005, rapport d'opération, InrapSRA Basse-Normandie, 3 vol.

Guillot B. (dir.), 2015 (à paraître), Forges médiévales et écurie de la Renaissance au château de Caen. Fouilles de 2005 (secteur nord-ouest), Monographie de la fouille de 2005, à paraître en 2015 aux Presses universitaires de Caen.

Grison F., 1615, L'escuirie du S. Federic Grison, gentilhomme napolitain. En laquelle est monstré l'ordre et l'art de choysir, donter, picquer, dresser et manier les chevaux tant pour l'usage de la guerre, qu'autre commodité de l'homme. Avec figures de diverses sortes de mors de bride. Nagueres traduicte d'italien en françois et nouvellement reveuë et augmentée, Paris, Adrian Périer.

LIÉVAUX P., 2005, Les écuries des châteaux français, Paris, Éditions du patrimoine, $303 \mathrm{p}$.
MÜNZBERG E., 2010, « Les écuries princières associant armureries et "kunstkammer". Un type de bâtiment de représentation destiné aux collections vers 1600 ", in LiÉvaux P., Franchet D'Espèrey P. (dir.), Architecture équestre. Hauts lieux dédiés au cheval en Europe, Arles, Actes Sud (« Arts Équestres »), p. 61-73.

Prentout H., 1904, La prise de Caen par Édouard III, 1346, étude critique, Caen, H. Delesques, 72 p. (extrait des Mémoires de l'Académie nationale des sciences, arts et belles-lettres de Caen).

Roche D., 2005, « Préface », in LiÉvaux P., Les écuries des châteaux français, Paris, Éditions du patrimoine, p. 9-17.

Salamagne A., 2010, « Les écuries dans le château médiéval », in LIÉVAuX P., FrANCHET D'ESPÈrEy P. (dir.), Architecture équestre. Hauts lieux dédiés au cheval en Europe, Arles, Actes Sud («Arts Équestres»), p. 23-37.

SCHWIEN J.-J. ET JEANNIN Y., 2012, « Loger, nourrir, équiper le cheval : un essai de synthèse pour la seconde partie du Moyen Âge dans l'est de la France et ailleurs ", in LAZARIS S. (dir.), Le cheval dans les sociétés antiques et médiévales, actes des journées d'étude internationales organisées par l'UMR 704.4 (étude des civilisations de l'Antiquité), Strasbourg, 6-7 novembre 2009, Turnhout, Brepols («Bibliothèque de l'Antiquité tardive », 22), p. 113-131, figures p. 240-271.

TACQUet J., 1614, «Philippica » ou haras de chevaux, Anvers, R. Bruneau, 284 p. 